\title{
PARÁMETROS DE VARIABILIDAD GENÉTICA DE BOVINOS CERTIFICADOS DE LA RAZA HOLSTEIN DEL CANTÓN CUENCA, ECUADOR
}

\section{GENETIC VARIABILITY PARAMETERS OF CERTIFIED HOLSTEIN CATTLE IN CUENCA DISTRICT, ECUADOR}

\author{
Silvana Méndez ${ }^{1,3}$, Manuel E. Soria ${ }^{1,3}$, Estuardo Palacios ${ }^{3, *}$, Omar Andrade ${ }^{3}$, Jorge Bustamante ${ }^{3}$, \\ José Luis Pesantez ${ }^{3}$, Juan Vázquez ${ }^{3}$, Guillermo Guevara ${ }^{3}$, y Antonio J. Vallecillo ${ }^{2,3}$
}

\footnotetext{
${ }^{1}$ Laboratorio de Biotecnología de la Reproducción Animal, Facultad de Ciencias Agropecuarias, Universidad de Cuenca, Granja Irquis, km 23, vía Cuenca-Girón-Pasaje, Parroquia Victoria del Portete, Ecuador.

${ }^{2}$ Laboratorio de Biología Molecular, Facultad de Ciencias Agropecuarias, Universidad de Cuenca, Av. 12 de octubre y Diego de Tapia, Cuenca, Ecuador. C.P. 010220.

${ }^{3}$ Escuela de Medicina Veterinaria y Zootecnia, Facultad de Ciencias Agropecuarias, Universidad de Cuenca, Av. 12 de octubre y Diego de Tapia, Cuenca, Ecuador. C.P. 010220.

* Autor para correspondencia E-mail: estuardo.palacios@ucuenca.edu.ec
}

\section{RESUMEN}

La raza Holstein es la que más contribuye a la producción láctea en Ecuador. Sin embargo, no existen datos de parámetros genéticos que describan las características de los hatos lecheros del país. Por ello, el objetivo de este trabajo fue analizar la variabilidad genética de bovinos registrados de la raza Holstein en el cantón Cuenca, Ecuador. Para ello se analizaron los marcadores microsatélites CSRM60, INRA083, CSSM66, ETH3, HEL9, TGLA53, BM1818, LSTS006, BM2113, ETH225, TGLA122, ETH10, TGLA227, INRA032 y SPS115 en 52 bovinos, de acuerdo a lo sugerido por la FAO para la caracterización genética de poblaciones de animales domésticos. Se encontró en promedio $8,4 \pm 1,88$ alelos y una frecuencia de $0,08 \pm 0,056$ de alelos nulos. Los valores de heterocigosidad observada y esperada alcanzaron $0,68 \pm 0,13$ y $0,82 \pm 0,07$, respectivamente. El contenido de información polimórfica osciló entre 0,70 y 0,88 , y el coeficiente de endogamia varió entre $-0,38$ y 0,45 . De los 15 locis analizados, solamente el CSSM66 presentó equilibrio Hardy-Weinberg, y 4 de ellos mostraron un alto nivel de endogamia. Se concluye que los loci estudiados muestran una alta variabilidad genética y alto polimorfismo. Esto ofrece amplia información para los estudios de paternidad e identidad. No obstante, de acuerdo al nivel de consanguinidad debe considerarse la selección de una alta diversidad de toros para los programas de inseminación artificial.

Palabras clave: microsatélites, alelos, heterocigosidad, índice de endogamia.

\section{ABSTRACT}

Holstein cattle account for most of the dairy production in Ecuador. However, there are no data on genetic parameters that describe the characteristics of the country's dairy herds. Therefore, the objective of this study was to analyze the genetic variability of registered Holstein cattle in Cuenca district, Ecuador. For this, the microsatellite markers CSRM60, INRA083, CSSM66, ETH3, HEL9, TGLA53, BM1818, LSTS006, BM2113, ETH225, TGLA122, ETH10, TGLA227, INRA032 and SPS115 were analyzed in 52 bovines, according to the guidelines on genetic characterization of domestic animal species suggested by FAO. It was found an average of $8.4 \pm 1.88$ alleles and a frequency of

Recibido: 10 julio 2019. Aceptado: 08 octubre 2019. 
$0.08 \pm 0.056$ of null alleles. The observed and expected heterozygosity values reached $0.68 \pm 0.13$ and $0.82 \pm 0.07$, respectively. The polymorphic information content ranged between 0.70 and 0.88 , while the inbreeding coefficient varied between -0.38 and 0.45 . Of the 15 loci analyzed, only CSSM66 had a Hardy-Weinberg equilibrium and 4 of them showed a high level of inbreeding. It is concluded that the loci studied show high genetic variability and polymorphism. This provides ample information for paternity and identity studies. However, according to the consanguinity level, high genetic diversity should be considered when selecting bulls for artificial insemination programs.

Key words: microsatellites, alleles, heterozygosity, inbreeding index.

\section{INTRODUCCIÓN}

La raza Holstein se ha extendido en todo el mundo debido a su enorme potencial lechero, al marketing, la venta de semen, embriones y animales; sin embargo, los genomas que se encuentran en cada región pueden provenir de un número limitado de toros (sementales); por lo que es de vital importancia controlar la endogamia y mantener la variabilidad genética en los hatos (Ocampo y Cardona, 2013). Situación que no difiere en Ecuador, ni en el Azuay, una de las 4 provincias con mayor producción láctea del país; en donde la raza Holstein registrada y sin registro representa un alto porcentaje de la ganadería lechera, solamente en el cantón Cuenca se estima una presencia del $89 \%$ de las razas existentes (Alvarado et al., 2016).

Referencias de estudios genéticos en diferentes países indican el empleo de marcadores microsatélites para la caracterización de la raza Holstein (Hanslik et al., 2000; Řehout et al., 2006; Ozkan et al., 2009; Rincón et al., 2013); sin embargo, en Ecuador se desconocen datos actuales sobre la variabilidad génica de esta raza. Se ha reportado que presenta problemas de consanguinidad y deficiencias reproductivas graves (Heins et al., 2006; Lucy, 2007; Galarza et al., 2017); por lo que, es necesario conocer las características de la estructura genética mediante un monitoreo sistemático del flujo de genes de la población Holstein presente en las zonas de mayor producción lechera del Ecuador.

Por lo anterior, este trabajo se orienta al análisis de marcadores genéticos microsatélites, a fin de estimar parámetros de variabilidad genética en bovinos registrados de la raza Holstein en hatos localizados en el cantón Cuenca, provincia del Azuay, Ecuador.

\section{MATERIALES Y MÉTODOS}

Se emplearon 52 bovinos Holstein registrados en la Asociación Holstein Freisian del Ecuador, lo que está de acuerdo con las recomendaciones de la FAO (2011) donde se considera un número de 30 a 50 animales para este tipo de estudio. Se colectaron 5 a $10 \mathrm{~mL}$ de sangre periférica por punción de la vena yugular o coccígea media con el sistema Vacuntainer $^{\circledR}$ en tubos con EDTA. Los animales correspondieron a una parte de la población de 5 rebaños, número representativo ya que el estudio se enfocó en la población de bovino Holstein que se encuentran registrados y que residen dentro de la provincia del Azuay. El número de animales muestreados dentro de cada hato respondió a la disponibilidad de los animales y la apertura de los dueños para que fuesen incluidos en el estudio. El incremento en el número de muestras implicaría introducir en el estudio animales sin registro y con el riesgo de obtener otra caracterización. Los hatos están localizados en el cantón Cuenca, provincia del Azuay, Ecuador, entre las coordenadas $2^{\circ} 39^{\prime}$ a $3^{\circ} 00^{\prime}$ de latitud Sur y $78^{\circ} 54^{\prime}$ a $79^{\circ} 26^{\prime}$ de longitud Oeste, con una altura aproximada de 2.500 m.s.n.m.

Cada una de las muestras colectadas e identificadas se transportaron en refrigeración $\left(4^{\circ} \mathrm{C}\right)$, protegidas de la luz solar para su conservación, hasta el laboratorio de Biología Molecular de la Facultad de Ciencias Agropecuarias, donde fueron procesadas para la obtención del ADN total.

Para la purificación del material genético a partir de $1 \mathrm{~mL}$ de la sangre se usó el kit comercial PureLink $^{\mathrm{TM}}$ Genomic DNA Mini Kit (Invitrogen, Carlsbad, California, USA) siguiendo las instrucciones del fabricante. Se determinó a cada una de las muestras de ADN total obtenida la concentración mediante espectrofometría (Epoch microplate spectrophotometer, Take3 microvolume plate (Biotek, Winooski, Vermont, USA). El ADN obtenido se empleó para la amplificación mediante PCR de 15 marcadores genéticos basados en microsatélites, seleccionados de los 30 sugeridos por la FAO para la caracterización genética de poblaciones bovinas (FAO, 2011). La selección se realizó basada en una revisión de bibliografía en la que se reporta el uso de estos marcadores, para así generar datos genéticos comparables con los previamente reportados (Armstrong et al., 2006; Piñeira et al., 2011; Delgado et al., 2012; Martínez et al., 2012; Pereira et al., 2012; Ginja et al., 2013). Los 15 marcadores genéticos fueron amplificados en 4 mezclas de reacciones de PCR de $10 \mu \mathrm{L}(20 \mathrm{mM}$ de Tris- $\mathrm{HCl}, \mathrm{pH} 8,4 ; 50 \mathrm{mM}$ de $\mathrm{KCl}, 1,5 \mathrm{mM}$ de $\mathrm{MgCl}_{2} ; 0,2 \mathrm{mM}$ de cada uno de los dNTP's; 0,1 $\mu \mathrm{M}$ 
de cada uno de los respectivos oligonucleótidos; 50 ng de ADN total; $0.5 \mathrm{U}$ de ADN polimerasa Taq), en la mezcla número 1 se incluyeron los oligonucleótidos para amplificar los marcadores CSRM60, INRA063 y CSSM66, en la mezcla 2, ETH3, HEL9, TGLA53, BM1818 y ILSTS006, en la mezcla 3, BM2113, ETH225, TGLA122 y ETH10, y en la mezcla 4, TGLA227, INRA032 y SPS115. Los detalles metodológicos asociados al análisis para la validación de los resultados, mezclas para el marcado fluorescente de cada uno de los productos de PCR correspondientes a los marcadores genéticos están descritos en la publicación de FAO (2011). Para cada una de las mezclas los parámetros de los perfiles de amplificación fueron optimizados en un termociclador (Mastercycler ${ }^{\circledR}$ nexus, Eppendorf, Hamburg, Germany). La optimización de parámetros se realizó mediante una curva de temperatura de alineamiento o hibridación de la mezcla de los primers. La verificación de los productos obtenidos fue mediante electroforesis en gel de agarosa. Una vez que se generaron los amplicones se analizaron cada uno de los marcadores genéticos mediante el uso del analizador genético ABI PRISM 3100 Genetic Analyzer (Applied Biosystems, Foster City, California, USA) (Identigen CIA. LTDA.).

Una vez generados los datos de cada uno de los alelos de los 15 marcadores genéticos, se analizaron para obtener las estimaciones de los valores correspondientes a parámetros genéticos.

Los parámetros genéticos poblacionales estimados fueron: número total de alelos por marcador microsatélite $(\mathrm{Na})$, frecuencia de alelos nulos (fNul) mediante el programa Genepop v.4.5 (Rousset, 2008). Dentro de parámetros de variación genética se estimaron las frecuencias alélicas, heterocigosis observada (Ho) y esperada (He), y el contenido de información polimórfica (CIP) mediante el programa Cervus v.3.03 (Kalinowski et al., 2007). Adicionalmente, el coeficiente de endogamia (Fis) y una evaluación del cumplimiento del supuesto de equilibrio HardyWeinberg a partir de una prueba exacta basada en cadenas markovianas (100 simulaciones con 5.000 iteraciones) mediante el programa Genepop v.4.5 (Rousset, 2008).

\section{RESULTADOS Y DISCUSIÓN}

Parámetros descriptivos de los marcadores microsatélites

Los valores de los principales parámetros genéticos para cada uno de los 15 marcadores analizados con sus respectivos promedios se presentan en la Tabla 1. Al comparar lo obtenido en este trabajo con lo reportado por Riojas et al. (2009), quienes evaluaron 8 microsatélites en

Tabla 1. Parámetros descriptivos de los 15 loci marcadores microsatélites analizados en los bovinos de raza Holstein $(n=52)$ en Cuenca, Ecuador.

Table 1. Descriptive parameters of the 15 microsatellite marker loci analyzed in Holstein cattle $(\mathrm{n}=$ 52), Cuenca, Ecuador.

\begin{tabular}{lcc}
\hline Marcador & Na & $f$ Nul \\
\hline CSRM60 & 6 & 0,00 \\
CSSM66 & 7 & 0,04 \\
INRA083 & 7 & 0,13 \\
ETH3 & 12 & 0,14 \\
BM1818 & 9 & 0,09 \\
TGLA53 & 9 & 0,00 \\
HEL9 & 8 & 0,05 \\
LSTS006 & 8 & 0,11 \\
TGLA122 & 11 & 0,14 \\
BM2113 & 11 & 0,14 \\
ETH10 & 8 & 0,20 \\
SPS115 & 6 & 0,00 \\
TGLA227 & 6 & 0,00 \\
INRA032 & 9 & 0,03 \\
ETH225 & 9 & 0,08 \\
PROMEDIO $( \pm$ EE) & $8,4( \pm 1,88)$ & $0,08( \pm 0,05)$ \\
\hline
\end{tabular}

Na: Número total de alelos por locus marcador microsatélite.

fNul: Frecuencia estimada de alelos nulos.

EE: Error estándar. 
39 bovinos Holstein residentes en el nordeste de México, se observó que el número promedio (Na) fue menor en 4 alelos. Por otro lado, Garza (2002) analizó 13 marcadores genéticos en Nuevo León encontrando en promedio 3 alelos menos a lo descrito en el presente trabajo. Otros investigadores, como Rahimi et al. (2006) en Irán, analizaron 13 marcadores genéticos, y Zhang et al. (2010) en China, 17 marcadores, y ambos autores encontraron un promedio de 9 alelos; mientras que Mejía et al. (2015) encontraron 10 alelos en 11 locus.

Puntualmente, los marcadores genéticos incluidos en el presente análisis que mostraron menor número de alelos fueron CSM60, SPS115 y el TGL227; en los que se encontró un mayor número de alelos fueron ETH3, TGLA122 y BM2113, este último resultado coincide con el previamente descrito por Garza (2002), Rahimi et al. (2006). En el caso del mayor número de alelos observados fue el marcador TGLA122 que coincide con lo informado por Mejía et al. (2015).

\section{Parámetros de variación genética}

Los valores de los parámetros de diversidad genética de los loci estudiados se muestran en la Tabla 2. Se encontró que la Ho fue inferior en promedio a la $\mathrm{He}$, similar a lo reportado por
Zhang et al. (2010), sin embargo, esto difiere de lo informado por Rahimi et al. (2006) y por Mejía et al. (2015), cuyas Ho mostraron valores mayores a los de la He. Como se observa en la Tabla 2, únicamente los loci CSRM60 y SPS115 mostraron una Ho con valores mayores a los de la $\mathrm{He}$, por tanto, estos loci con mayor valor muestran gran heterocigosis. Riojas et al. (2009) reportaron Ho con cifras muy variables, desde 0,41 para el locus SPS115, que en este trabajo fue superior en 0,47 $(\mathrm{Ho}=0,88)$, hasta valores como 0,83 en el BM2113, una cifra superior a lo encontrado en este trabajo en $0,25(\mathrm{Ho}=0,58)$. En los restantes loci de este trabajo, He supera a Ho en más de 0,10 lo cual expresa una desviación de los apareamientos aleatorios y mayor variabilidad genética (Martínez et al., 2015).

Por otro lado, el contenido de información polimórfica (CIP) resultó alto en todos los loci comparado con el valor indicado como CIP informativo $(>0,50)$ por Martínez et al. (2015) y Mejía et al. (2015), valor que reafirma una variabilidad genética alta. Los loci con menor CIP: INRA083 y SPS115, presentaron valores de 0,70, lo que es un indicativo de la utilidad para estudios de paternidad, que en su esencia requieren de varios loci polimórficos o informativos, de tal manera que la probabilidad de inclusión o exclusión garanticen

Tabla 2. Parámetros de variación genética, heterocigosidad observada (Ho) y esperada (He), contenido de información polimórfica (CIP), coeficiente de endogamia (Fis) y equilibrio Hardy-Weinberg (EHW) de cada uno de los 15 loci marcadores microsatélites estudiados.

Table 2. Parameters of genetic variation, observed (Ho) and expected ( $\mathrm{He}$ ) heterozygosity, polymorphic information content (CIP), inbreeding coefficient (Fis) and Hardy-Weinberg equilibrium (EHW) of each of the 15 microsatellite marker loci studied.

\begin{tabular}{lllcrc}
\hline Marcador & Ho & He & CIP & Fis & EHW \\
\hline CSRM60 & 0,82 & 0,77 & 0,79 & $-0,06$ & 0,00 \\
INRA083 & 0,55 & 0,72 & 0,70 & 0,25 & 0,00 \\
CSSM66 & 0,77 & 0,81 & 0,81 & 0,05 & 0,21 \\
ETH3 & 0,52 & 0,90 & 0,86 & 0,43 & 0,00 \\
HEL9 & 0,67 & 0,82 & 0,79 & 0,19 & 0,00 \\
TGLA53 & 0,81 & 0,86 & 0,82 & 0,07 & 0,00 \\
BM1818 & 0,76 & 0,84 & 0,84 & 0,10 & 0,00 \\
LSTS006 & 0,61 & 0,85 & 0,81 & 0,28 & 0,00 \\
BM2113 & 0,58 & 0,89 & 0,88 & 0,36 & 0,00 \\
ETH225 & 0,73 & 0,84 & 0,83 & 0,14 & 0,00 \\
TGLA122 & 0,56 & 0,88 & 0,88 & 0,38 & 0,00 \\
ETH10 & 0,43 & 0,78 & 0,82 & 0,45 & 0,00 \\
TGLA227 & 0,70 & 0,80 & 0,74 & 0,13 & 0,00 \\
INRA032 & 0,76 & 0,87 & 0,81 & 0,14 & 0,00 \\
SPS115 & 0,88 & 0,63 & 0,70 & $-0,38$ & 0,00 \\
PROMEDIO ( \pm EE) & $0,68( \pm 0,13)$ & $0,82( \pm 0,07)$ & - & - & - \\
\hline
\end{tabular}

EE: Error estándar. 
seguridad de lo reportado. De manera general el CIP promedio fue mayor que el encontrado por Zhang et al. (2010) y Mejía et al. (2015).

Este parámetro positivo indica que en la actualidad hay un amplio polimorfismo $y$ diversidad en los animales de los hatos evaluados de la raza Holstein del cantón Cuenca, situación que debe mantenerse por las autoridades, productores y los especialistas para continuar garantizando el uso de una amplia gama de toros, y así evitar que las nuevas generaciones de bovinos de esta raza provengan de un grupo reducido de reproductores.

En el 33\% de los loci estudiados se encontró valores muy altos del coeficiente de endogamia (Fis), otros loci se observan valores moderados de este coeficiente. Valores superiores a 0,35 de Fis se observan en marcadores ETH3, ETH10, TGLA122 y BM2113, lo cual refleja un nivel muy alto de homocigotos, ya que según Martínez (2008) un Fis $>0,25$ ya se considera alto. Esto señala una deriva en esos loci, contrariamente, en el locus CSRM60 y principalmente en el locus SPS115 el Fis exhibe valores negativos lo cual indica mayores niveles de heterocigotos. En Colombia, Mejía et al. (2015) obtuvieron resultados contrarios, excepto en el locus TGLA53 $(0,66)$, pero en los loci ETH3, ETH10, ETH225, BM2113, SPS115, TGLA122 y TGLA227 encontraron para la raza Holstein valores para el Fis muy bajos. Resultados similares fueron reportados por Martínez et al. (2015) en Costa Rica, al igual que lo encontrado por Egito et al. (2007) en Brazil, en donde el valor de Fis fue de 0,075 ; lo que indica que se utilizan toros diferentes en la reproducción lo cual influye en que todos los marcadores genéticos presenten equilibrio Hardy-Weinberg. En este estudio los resultados observados en los mismos loci fueron diferentes ya que solamente el marcador CSSM66 se presentó en equilibrio.

Los loci CSRM60, SPSS115, TGLA53 y TGLA227 no presentaron alelos nulos y para el caso de los loci INRA032, HEL9 y BM1818 se encontraron frecuencias cercanas a cero. La más alta se encontró en el marcador ETH10, y el resto de los loci tuvieron frecuencias con valores relativamente altos, lo que pudiera relacionarse con la diferencia respecto a los heterocigotos esperados. Además, puede ser causa de desviaciones significativas del equilibrio HardyWeinberg. Otros factores que afectan el equilibrio Hardy-Weinberg pueden ser el alto nivel del Fis encontrado en cuatro loci, niveles de moderado a alto del Fis en otros seis loci. La frecuencia general de alelos nulos de 0,08 fue más alta que la referida por Mejía et al. (2015), lo que pudiera relacionarse con la deficiencia de Ho en loci como TGLA122, ETH10, BM2113 y ETH3, Fis alto y que haya una desviación del equilibrio en la mayoría de los loci.

\section{CONCLUSIONES}

Los resultados del análisis genético realizado a los 52 bovinos presentes en 5 hatos del cantón Cuenca permiten tener una descripción actual del estado de un grupo numeroso de loci, de los recomendados por la comunidad científica, resultados que muestran una alta variabilidad genética y alto polimorfismo en los bovinos Holstein estudiados. Esto ofrece amplia información para la realización de estudios de análisis de paternidad e identidad. No obstante, debe seleccionarse toros con mayor diversidad para los programas de inseminación artificial dado que se encontró que 4 de los 15 loci estudiados presentaron un alto nivel de endogamia.

\section{AGRADECIMIENTOS}

Los autores agradecen a la Dirección de Investigación de la Universidad de Cuenca (DIUC) por el financiamiento para la ejecución de este proyecto (Convocatoria XIV) y a la Asociación Holstein Freisian Filial Cuenca, por las facilidades prestadas para la toma de las muestras.

\section{LITERATURA CITADA}

Alvarado, J. M.C., A.C.B. Rodas, y L.G. Ayala. 2016. Características morfométricas e índices zoométricos de los grupos raciales bovinos existentes en el Cantón Cuenca. Tesis para obtener el grado de Médico Veterinario. Disponible en http://dspace.ucuenca.edu. ec/handle/123456789/25281 (Consultado 31mayo-2019)

Armstrong, E., A. Postiglioni, A. Martínez, G. Rincón, and J.L. Vega-Pla. 2006. Microsatellite analysis of a sample of Uruguayan Creole bulls (Bos taurus). Genet. Mol. Biol. 29(2):267272. doi:10.1590/S1415-47572006000200012.

Delgado, J.V., A.M. Martínez, A. Acosta, L.A. Alvarez, E. Armstrong, E. Camacho, et al. 2012. Genetic characterization of LatinAmerican Creole cattle using microsatellite markers. Anim. Genet. 43(1):2-10. doi: 10.1111/j.1365-2052.2011.02207.x.

Egito, A., S.R. Paiva, M.doS. Albuquerque, L.D.A. Mariante, S.R. Castro, y D. Grattapaglia. 2007. Microsatellite based genetic diversity and relationships among ten Creole and commercial cattle breeds raised in Brazil. BMC Genetics. 8:83 doi:10.1186/1471-2156-8-83.

FAO. 2011. Molecular genetic characterization of animal genetic resources. Food and Agriculture Organization of the United Nations (FAO). Animal Production and Health. Guidelines 9. Rome, Italia. 
Galarza A., L.R., F.P. Perea G., R.V. Guevara V., J.P. Alvarado M. y D.E. Argudo G. 2017. Caracterización de la fertilidad en un rebaño Holstein Neozelandés de la sierra Sur del Ecuador. Maskana 8:113-116.

Garza L., J.M. 2002. Determinación del número de variantes $y$ frecuencias génicas de microsatélites de ADN en las razas bovinas Holstein Friesian y Simmental del noreste de México. Tesis de Maestría en Medicina Veterinaria. Universidad Autónoma de Nuevo León, San Nicolás de los Garza, Nuevo León, México.

Ginja, C, L.T. Gama, O. Cortes, J.V. Delgado, S. Dunner, D. García, et al. 2013. Analysis of conservation priorities of Iberoamerican cattle based on autosomal microsatellite markers. Genet Sel. Evol. 45:35. doi: 10.1186/1297-968645-35.

Hanslik, S., B. Harr, G. Brem, y C. Schlötterer. 2000. Microsatellite analysis reveals substantial genetic differentiation between contemporary New World and Old World Holstein Friesian populations. Anim. Genet. 31(1):31-18. doi: 10.1046/j.1365-2052.2000.00569.x.

Heins, B.J., L.B. Hansen, y A.J. Seykora. 2006. Fertility and survival of pure Holsteins versus crossbreds of Holstein with Normande, Montbeliarde, and Scandinavian Red. J Dairy Sci. 89(12):4944-4951. doi: 10.3168/jds.S00220302(06)72545-0.

Kalinowski, S.T., M.L. Taper, y T.C. Marshall. 2007. Revising how the computer program CERVUS accommodates genotyping error increases success in paternity assignment. Mol. Ecol. 16(5):1099-1106. doi: 10.1111/j.1365294X.2007.03089.x

Lucy, M.C. 2007. Fertility in high-producing dairy cows: reasons for decline and corrective strategies for sustainable improvement. Soc. Reprod. Fertil. Suppl. 64:237-254.

Martínez, A.M., L.T. Gama, J. Cañón, C. Ginja, J.V. Delgado, S. Dunner, et al. 2012. Genetic footprints of Iberian cattle in America 500 years after the arrival of Columbus. PLoS One. 7(11):e49066. doi: 10.1371/journal. pone.0049066.

Martínez, M., B. Vargas, J.M. Cordero, I. Chacón, y B. León. 2015. Diversidad genética entre subpoblaciones raciales bovinas de Costa Rica. Agronomía Costarricense 39(2):33-45.

Mejía, L.G., R.A. Hernández, C.Y. Rosero, y C.E. Solarte. 2015. Análisis de la diversidad genética de ganado bovino lechero del trópico alto de Nariño mediante marcadores moleculares heterólogos de tipo microsatélite. Rev. Med. Vet. Zoot. 62(3):18-33. doi: 10.15446/rfmvz. v62n3.54938.
Ocampo, G.R., y Cardona, C.H. 2013. La endogamia en la producción animal. Rev. Colombiana Cienc. Anim. 5(1):463-479.

Ozkan, E., M.I. Soysal, M. Ozder, E. Koban, O. Sahin, y I. Togan. 2009. Evaluation of parentage testing in the Turkish Holstein population based on 12 microsatellite loci. Livest. Sci. 124(1-3):101-106. doi: 10.1016/j. livsci.2009.01.004

Pereira, J.A.C., M.H. Carino, R. Hoyos, A. RogbergMuñoz, A. Loza, J.P. Liron, et al. 2012. Diseño de un programa de conservación de un hato de Criollo Yacumeño asistido por marcadores genéticos en Santa Cruz-Bolivia. AICA 2(2012):155-159.

Piñeira, V., J., F. Mujíca C., R. Felmer D., M, Ortiz L., G, Pizarro I. y M. Aracena N. 2011. Caracterización genética de un rebaño de bovino criollo patagónico chileno. Agro Sur 39(1):46-56.

Rahimi, G., A. Nejati-Javaremi, D. Saneei, y K. Olek. 2006. Estimation of genetic variation in Holstein young bulls of Iran AI Station using molecular markers. Asian-Australas. J. Anim. Sci. 19(4):463-467. doi: doi.org/10.5713/ ajas.2006.463

Řehout, V., E. Hradecká, y J. Čítek. 2006. Evaluation of parentage testing in the Czech population of Holstein cattle. Czech. J. Anim. Sci. 51(12):503509. doi: 10.17221/3970-CJAS.

Rincón, F.A.J., H.A. López, y Z.J. Echeverri. 2013. Estructura y diversidad genética en vacas Holstein de Antioquia usando un polimorfismo del gen bGH. Rev. MVZ Cordoba. 18(1):33463354. doi: 10.21897/rmvz.197

Riojas-Valdes, V.M., J.C. Gomez-de-la-Fuente, J.M. Garza-Lozano, D.C. Gallardo-Blanco, J.N. De Tellitu-Schutz, A. Wong-Gonzalez, et al. 2009. Exclusion probabilities of 8 DNA microsatellites in 6 cattle breeds from Northeast Mexico. J. Anim. Vet. Adv. 8(1):6266.

Rousset, F. 2008. Genepop'007: a complete reimplementation of the genepop software for Windows and Linux. Mol. Ecol. Resour. 8(1):103-106. doi: 10.1111/j.14718286.2007.01931.x.

Zhang, Y., Y. Wang, D. Sun., Y. Yu, y Y. Zhang. 2010. Validation of 17 microsatellite markers for parentage verification and identity test in Chinese Holstein cattle. Asian-Australas. J. Anim. Sci. 23(4):425-429. doi: 10.5713/ ajas.2010.90424. 